\author{
Wladyslaw Koc \\ prof. dr hab. inż. \\ Politechnika Gdańska \\ Katedra Transportu Szynowego i Mostów \\ kocwl@pg.gda.pl
}

DOI: $10.35117 /$ A_ENG_17_08_02

\title{
Modeling variable curvature on the turnout diverging track length
}

\begin{abstract}
The paper presents three variants of variable curvature application on the entire length of the turnout diverging track. One linear and two nonlinear curvature cases: described by the function of $\mathrm{C} 1$ and $\mathrm{C} 0$ class were considered. It turned out that both linear curve and the function of $\mathrm{C} 1$ class requires a significant (even twice) length of diverging track extending with respect to the base turnout. The curvature described by the function of class $\mathrm{C} 0$ proved to be far more favorable, although the necessary extension of the turnout is still considerable. In the turnouts with variable curvature of diverging track it is possible to shape the horizontal ordinates flexibly by manipulating the crossing angle and radius. The study shows the effects of such a procedure, the aim of which was to obtain an appropriate value of the final ordinate.
\end{abstract}

Keywords: Railway turnout; Modeling curvature; Horizontal ordinates analysis

\section{Introduction}

In the typical geometric configuration of the railway reverse track (ordinary), a single circular arc is used (no transition curves). Slant turnout $1: n$ uniquely determines the tangent angle at the end of the reverse track (for $l=l_{k}$ ). The curvature of a circular arc with a radius $R$ is constant over the entire length and equals $k=\frac{1}{R}$ (in $\mathrm{rad} / \mathrm{m}$ ), and angle of inclination expressed in radians $\Theta(l)=\int k(l) d l=k l, l \in\left\langle 0, l_{k}\right\rangle$. On this basis it is easy to determine the length of the classic reverse track:

$$
l_{k}=\frac{\operatorname{atan} \frac{1}{n}}{k}=R \operatorname{atan} \frac{1}{n}
$$

The length of the entire turnout is determined by the pattern

$$
a=2 R \tan \left(\frac{1}{2} \operatorname{atan} \frac{1}{n}\right)
$$

The given formulas concern the so-called unhampered turnout, not included in track connections. Most often, however, turnouts are used to connect parallel tracks, and then it is crucial to maintain the required distance between track axes. With the use of traditional turnouts (with turning in the form of a circular arc), the fulfillment of this condition often necessitates modifications. Track alignments have shortened end assemblies, which makes it possible to connect them directly to other turnouts. The magnitude of the shortening or elongation in the reverse path of the rolling tracks of the inner rail depends on the distance between the parallel track axes and the slunt turnout. The number of turnouts produced in both versions is limited, which makes geometric solutions for increased train speeds difficult.

At the same time, in the traditional solution there are places of violent, jumping change of the ordinate curve of the curvature at the beginning and end of turnout. Therefore, in recent years, in many countries, in order to smooth the curvature of these regions, "clitoris sections" 
on both sides of the circular arc, on which the curvature varies linearly $[2,5,6]$. In the paper [3] an in-depth kinematics analysis of selected turnouts was conducted [4]. It has been pointed out that in the case of standard turnouts, the change of the operating shift to the larger radius turnout in the reverse path usually results in the need to increase the track gauge. In the case of turnouts with linear curvature segments - due to the individual nature of their design - it is possible to adjust the given route to the local requirements.

However, it is important to note that this is not the only way to improve the disadvantage of classic travels. Therefore, the method should be approached in a methodical way, considering the various ways of solving the problem. At the same time, it is important to realize that any modification of the curvature of the curve in turnout, while retaining the existing slant, will increase the length of this path so that the angle value can be maintained. $\Theta\left(l_{k}\right)$. In this study will be presented three variants of the use of variable curvature (also nonlinear) over the entire length of the reverse turnout. To determine equations of curvature and corresponding parametric equations $x(l)$ i $y(l)$ the methodology described in the work will be used [1].

\section{Linear curvature over the entire length of the reverse track (Variant I)}

The first case considered is the use of linear curvature along the entire length of the reverse track, namely the replacement of the two circular arc curves of transition in the form of a clothoid.

For the interval $l \in\left\langle 0, \frac{l_{k}}{2}\right\rangle$ we accept conditions:

$$
\left\{\begin{array}{l}
k(0)=0 \\
k\left(\frac{l_{k}}{2}\right)=\frac{1}{R}
\end{array}\right.
$$

and differential equation

$$
k^{\prime \prime}(l)=0
$$

The solution to the differential problem (3), (4) is as follows:

$$
k(l)=\frac{2}{R l_{k}} l
$$

and the equation of angle of tangency has the form

$$
\Theta(l)=\frac{1}{R l_{k}} l^{2}
$$

For the interval $l \in\left\langle\frac{l_{k}}{2}, l_{k}\right\rangle$ there are conditions:

$$
\left\{\begin{array}{l}
k\left(\frac{l_{k}}{2}\right)=\frac{1}{R} \\
k\left(l_{k}\right)=0
\end{array}\right.
$$

and the differential equation (4). As a result of the solution of the differential problem (4), (7) we obtain: 
and

$$
k(l)=\frac{2}{R}-\frac{2}{R l_{k}} l
$$

$$
\Theta(l)=-\frac{l_{k}}{2 R}+\frac{2}{R} l-\frac{1}{R l_{k}} l^{2}
$$

At the endpoint of the reverse track, for $l=l_{k}$, The tangent angle is $\quad \Theta\left(l_{k}\right)=\frac{l_{k}}{2 R}$. This results in the required reverse track length

$$
l_{k}=2 R \operatorname{atan} \frac{1}{n}
$$

Figure 1 shows a graph of linear curvature over the entire length of the reverse turnout $\mathrm{Rz}$ $1: 14-760$. Applying this solution would require extension of the reverse track to $l_{k}=108,388$ $\mathrm{m}$, that is, doubling its length relative to the standard solution.

Elevations horizontal axis of the return track (Fig. 2) are referred to the corresponding parametric equations.

- The interval $l \in\left\langle 0, \frac{l_{k}}{2}\right\rangle$

$$
\begin{gathered}
x(l)=l-\frac{l^{5}}{10 R^{2} l_{k}^{2}} \\
y(l)=\frac{l^{3}}{3 R l_{k}} \quad \text { or } \quad y(l)=\frac{l^{3}}{3 R l_{k}}-\frac{l^{7}}{42 R^{3} l_{k}^{3}}
\end{gathered}
$$

- The interval $l \in\left\langle\frac{l_{k}}{2}, l_{k}\right\rangle$

$x(l)=$

$x\left(\frac{l_{k}}{2}\right)+\cos \left(\frac{l_{k}}{4 R}\right)\left(l-\frac{l_{k}}{2}\right)-\frac{1}{2 R} \sin \left(\frac{l_{k}}{4 R}\right)\left(l-\frac{l_{k}}{2}\right)^{2}-\frac{1}{6}\left[\frac{1}{R^{2}} \cos \left(\frac{l_{k}}{4 R}\right)-\frac{2}{R l_{k}} \sin \left(\frac{l_{k}}{4 R}\right)\right](l-$ $\left.\frac{l_{k}}{2}\right)^{3}+\frac{1}{24}\left[\frac{6}{R^{2} l_{k}} \cos \left(\frac{l_{k}}{4 R}\right)+\frac{1}{R^{3}} \sin \left(\frac{l_{k}}{4 R}\right)\right]\left(l-\frac{l_{k}}{2}\right)^{4}+$

$\frac{1}{120}\left[\left(\frac{1}{R^{4}}-\frac{12}{R^{2} l_{k}^{2}}\right) \cos A_{20}-\frac{12}{R^{3} l_{k}} \sin \left(\frac{l_{k}}{4 R}\right)\right]\left(l-\frac{l_{k}}{2}\right)^{5}$

$y(l)=$

$y\left(\frac{l_{k}}{2}\right)+\sin \left(\frac{l_{k}}{4 R}\right)\left(l-\frac{l_{k}}{2}\right)+\frac{1}{2 R} \cos \left(\frac{l_{k}}{4 R}\right)\left(l-\frac{l_{k}}{2}\right)^{2}-\frac{1}{6}\left[\frac{2}{R l_{k}} \cos \left(\frac{l_{k}}{4 R}\right)+\frac{1}{R^{2}} \sin \left(\frac{l_{k}}{4 R}\right)\right]\left(l-\frac{l_{k}}{2}\right)^{3}$

Applying a linear curvature over the entire length of the reverse track requires doubling its length, so doubling the length of the entire turnout. An alternative would be to maintain the 
same length by doubling the radius of the circular arc. The practical application of such a solution seems doubtful; there is still the possibility to correct the slant.

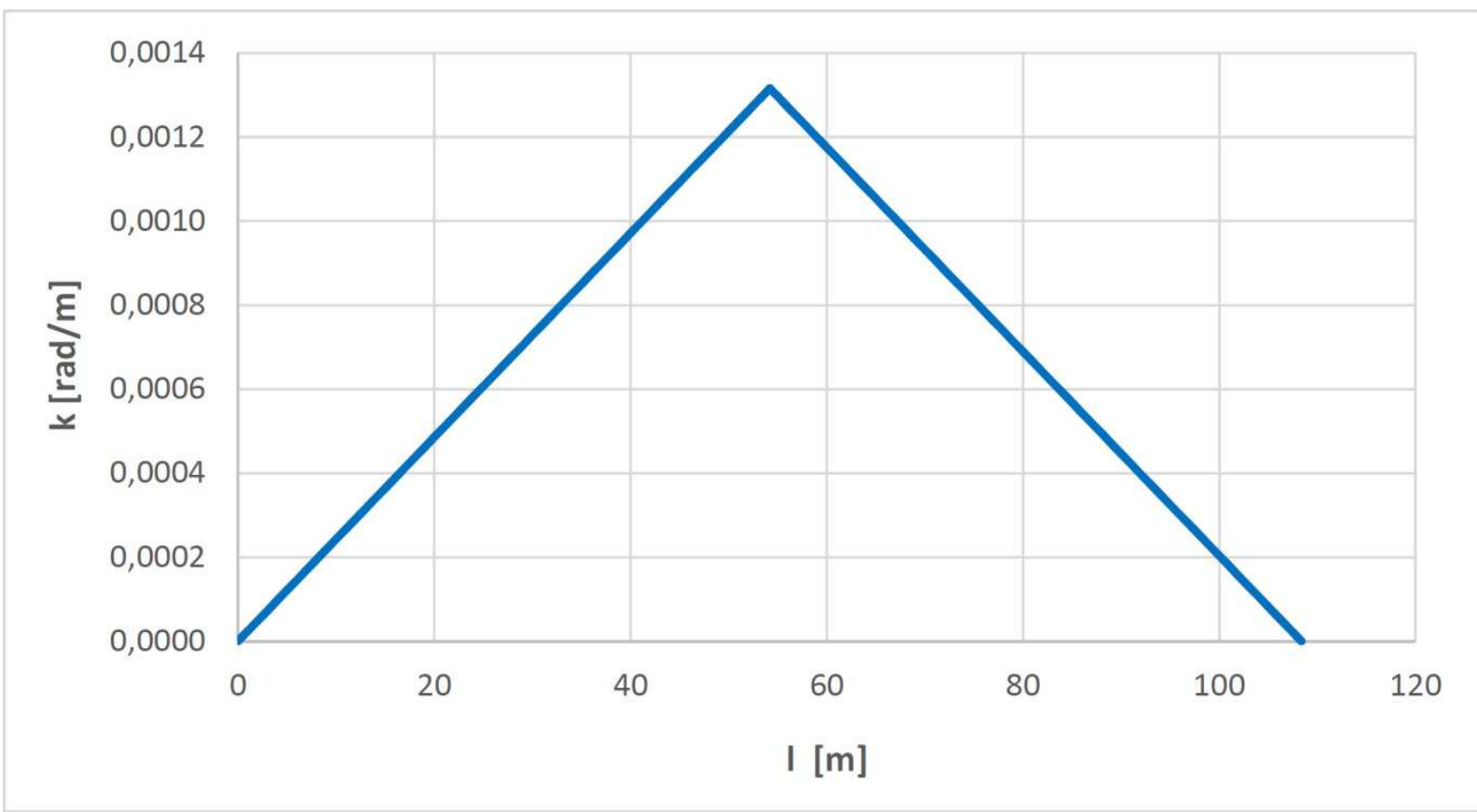

1. Graph of linear curvature along the entire length of the reverse turnout track Rz 1:14-760

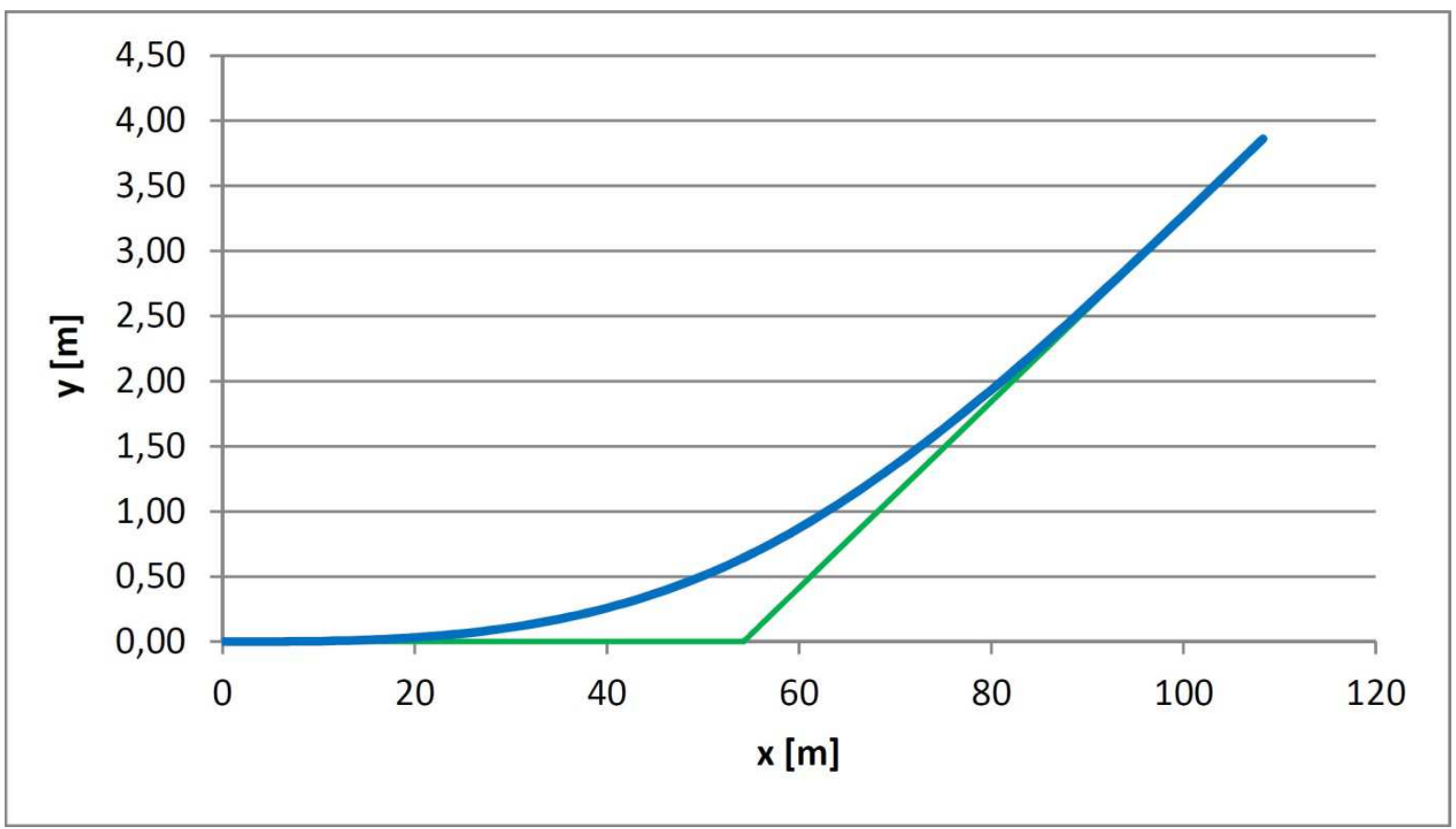

2. Graph of the function $y(x)$ for the reverse turnout track $\mathrm{Rz} 1: 14-760$ the curvature of the line (on a contaminated scale) 
Application of nonlinear curvature described by the class $\mathbf{C}^{\mathbf{1}}$ function over the entire length of the reverse track (Variant II)

Another case will be the application of the non-linear curvature along the entire length of the reverse track, specifically the replacement of a circular arc with two Bloss curves. This implies introducing the curvature described by the class $\mathrm{C}^{1}$ function.

Foe interval $l \in\left\langle 0, \frac{l_{k}}{2}\right\rangle$ we accept conditions:

$$
\left\{\begin{array}{l}
k(0)=k^{\prime}(0)=0 \\
k\left(\frac{l_{k}}{2}\right)=\frac{1}{R} \\
k^{\prime}\left(\frac{l_{k}}{2}\right)=0
\end{array}\right.
$$

and differential equation

$$
k^{(4)}(l)=0
$$

The solution to the differential problem (15), (16) is as follows:

$$
k(l)=\frac{12}{R l_{k}^{2}} l^{2}-\frac{16}{R l_{k}^{3}} l^{3}
$$

and the equation of angle of tangency has the form

$$
\Theta(l)=\frac{4}{R l_{k}^{2}} l^{3}-\frac{4}{R l_{k}^{3}} l^{4}
$$

For the interval $l \in\left\langle\frac{l_{k}}{2}, l_{k}\right\rangle$ there are conditions:

$$
\left\{\begin{array}{l}
k\left(\frac{l_{k}}{2}\right)=\frac{1}{R} \\
k^{\prime}\left(\frac{l_{k}}{2}\right)=0 \\
k\left(l_{k}\right)=k^{\prime}\left(l_{k}\right)=0
\end{array}\right.
$$

and the differential equation (12). As a result of the solution of the differential problem (16), (19) we obtain:

and

$$
k(l)=-\frac{4}{R}+\frac{24}{R l_{k}} l-\frac{36}{R l_{k}^{2}} l^{2}+\frac{16}{R l_{k}^{3}} l^{3}
$$

$$
\Theta(l)=\frac{l_{k}}{2 R}-\frac{4}{R} l+\frac{12}{R l_{k}} l^{2}-\frac{12}{R l_{k}^{2}} l^{3}+\frac{4}{R l_{k}^{3}} l^{4}
$$

As it turns out, the angle of incidence of the tangent at the end point, for $l=l_{k}$, is the same as in the case of linear curvature over the entire length of the reverse track, i.e. defined 
by the formula (10). In this situation, the required reverse track length must also be two times higher than the standard one, using a circular arc.

Figure 3 shows the graph of nonlinear curvature described by the class function $\mathrm{C}^{1}$ along the length of the reverse turnout $\mathrm{Rz} 1: 14-760$. As in case of variant $\mathrm{I}$, the use of such a solution would require extension of the reverse track to $l_{k}=108,388 \mathrm{~m}$.

The horizontal ordinates of the reverse track are described by parametric equations.

- The interval $l \in\left\langle 0, \frac{l_{k}}{2}\right\rangle$

$$
\begin{gathered}
x(l)=l-\frac{8}{7 R^{2} l_{k}^{4}} l^{7}+\frac{2}{R^{2} l_{k}^{5}} l^{8}-\frac{8}{9 R^{2} l_{k}^{6}} l^{9} \\
y(l)=\frac{1}{R l_{k}^{2}} l^{4}-\frac{4}{5 R l_{k}^{3}} l^{5}
\end{gathered}
$$

The interval $l \in\left\langle\frac{l_{k}}{2}, l_{k}\right\rangle$

$$
\begin{aligned}
& x(l)=x\left(\frac{l_{k}}{2}\right)+\cos \left(\frac{l_{k}}{4 R}\right)\left(l-\frac{l_{k}}{2}\right)-\frac{1}{2 R} \sin \left(\frac{l_{k}}{4 R}\right)\left(l-\frac{l_{k}}{2}\right)^{2}-\frac{1}{6 R^{2}} \cos \left(\frac{l_{k}}{4 R}\right)\left(l-\frac{l_{k}}{2}\right)^{3}+ \\
& \frac{1}{24}\left(\frac{1}{R^{3}}+\frac{24}{R l_{k}^{2}}\right) \sin \left(\frac{l_{k}}{4 R}\right)\left(l-\frac{l_{k}}{2}\right)^{4} \\
& y(l)=y\left(\frac{l_{k}}{2}\right)+\sin \left(\frac{l_{k}}{4 R}\right)\left(l-\frac{l_{k}}{2}\right)+\frac{1}{2 R} \cos \left(\frac{l_{k}}{4 R}\right)\left(l-\frac{l_{k}}{2}\right)^{2}-\frac{1}{6 R^{2}} \sin \left(\frac{l_{k}}{4 R}\right)\left(l-\frac{l_{k}}{2}\right)^{3}- \\
& \frac{1}{24}\left(\frac{1}{R^{3}}+\frac{24}{R l_{k}^{2}}\right) \cos \left(\frac{l_{k}}{4 R}\right)\left(l-\frac{l_{k}}{2}\right)^{4}
\end{aligned}
$$

Contrary to variant $\mathrm{I}$, the individual words of the patterns describing the coordinates $x(l) \mathrm{i}$ $y(l)$ in the interval $l \in\left\langle\frac{l_{k}}{2}, l_{k}\right\rangle$ only to a limited extent tend to disappear; This is especially true of functions $y(l)$. Therefore, it may be more advantageous to define horizontal ordinates by means of numerical integration.

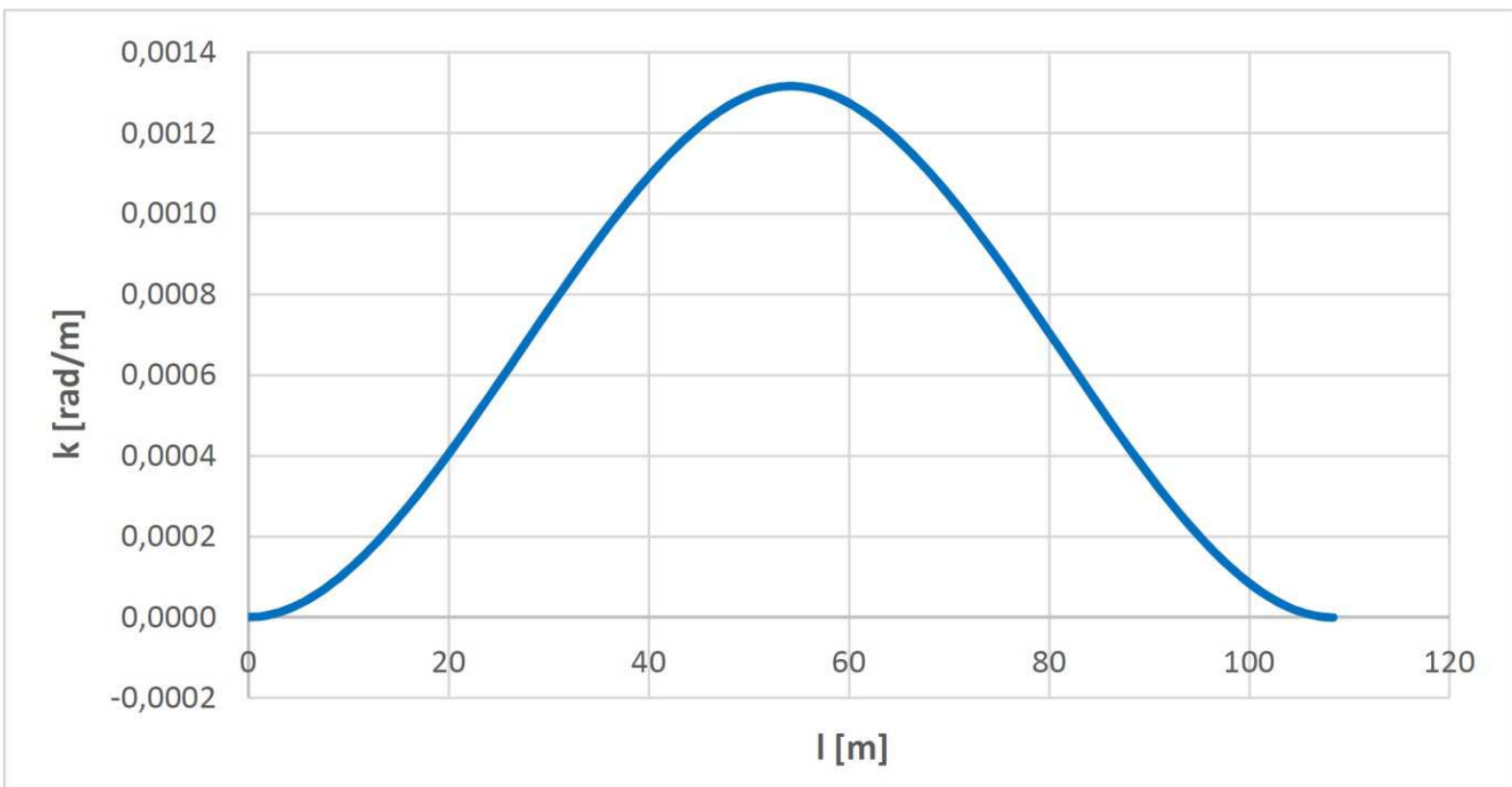

3. Graph of non-linear curves described by the class function $C^{1}$ along the entire length of the reverse turnout track Rz 1:14-760 


\section{Application of the non-linear curvature described by the class $C^{0}$ function over the entire length of the back path (Variant III)}

Another case will be the application of the non-linear curvature path described by the class $\mathrm{C}^{0}$ function along the entire length.

For the interval $l \in\left\langle 0, \frac{l_{k}}{2}\right\rangle$ we accept conditions:

$$
\begin{cases}k(0)=0 & k\left(\frac{l_{k}}{2}\right)=\frac{1}{R} \\ k^{\prime}(0)=\frac{C}{R l_{k}} & k^{\prime}\left(\frac{l_{k}}{2}\right)=0\end{cases}
$$

and the differential equation (16). We assume that the numerical coefficient $C \geq 0$. As a result of the solution of the differential problem (16), (26) we obtain

$$
k(l)=\frac{C}{R l_{k}} l+\frac{4(3-C)}{R l_{k}^{2}} l^{2}-\frac{4(4-C)}{R l_{k}^{3}} l^{3}
$$

The equation of the angle of incidence of the tangent has the form

$$
\Theta(l)=\frac{C}{2 R l_{k}} l^{2}+\frac{4(3-C)}{3 R l_{k}^{2}} l^{3}-\frac{4-C}{R l_{k}^{3}} l^{4}
$$

Obtaining a correct solution requires the adoption of a suitable value of the parameter $C$. Curvature function $k(l)$ must be a monotonous function, growing for $l>0$; hence the condition

$$
k^{\prime}(l)=\frac{C}{R l_{k}}+\frac{8(3-C)}{R l_{k}^{2}} l-\frac{12(4-C)}{R l_{k}^{3}} l^{2} \geq 0
$$

After entering a non-variable not appointed $\xi=\frac{l}{l_{k}}$ Condition (29) can be written as follows:

$$
C+8(3-C) \xi-12(4-C) \xi^{2} \geq 0
$$

At the same time the curvature cannot appear on the curvature graph, and the required upward curvature defines another condition

$$
k^{\prime \prime}(l)=\frac{8(3-C)}{R l_{k}^{2}}-\frac{24(4-C)}{R l_{k}^{3}} l \leq 0
$$

The boundary of the area is therefore a straight line, and the given condition must be fulfilled at both ends. After converting and entering a variable $\xi$ we obtain

$$
8(3-C)-24(4-C) \xi \leq 0 \quad \xi \in\left\langle 0, \frac{1}{2}\right\rangle
$$


At the starting point, for $\xi=0,8(3-C) \leq 0$, hence $C \geq 3$. At the end of the interval, for $\xi=\frac{1}{2}, 8(3-C)-12(4-C) \leq 0$, hence $C \leq 6$. It follows that parameter $\mathrm{C}$ must accept values from the interval $C \in\langle 3 ; 6\rangle$.

For the interval $l \in\left\langle\frac{l_{k}}{2}, l_{k}\right\rangle$ there are conditions:

$$
\left\{\begin{array}{rlrl}
k\left(\frac{l_{k}}{2}\right)=\frac{1}{R} & & \left(l_{k}\right)=0 \\
k^{\prime}\left(\frac{l_{k}}{2}\right)=0 & k^{\prime}\left(l_{k}\right)=-\frac{C}{R l_{k}}
\end{array}\right.
$$

and the differential equation (16). As a result of the solution of the differential problem (16), (31) we obtain:

and

$$
k(l)=-\frac{4-C}{R}+\frac{24-5 C}{R l_{k}} l-\frac{4(9-2 C)}{R l_{k}^{2}} l^{2}+\frac{4(4-C)}{R l_{k}^{3}} l^{3}
$$

$$
\Theta(l)=\frac{4-C}{8 R} l_{k}-\frac{4-C}{R} l+\frac{24-5 C}{2 R l_{k}} l^{2}-\frac{4(9-2 C)}{3 R l_{k}^{2}} l^{3}+\frac{4-C}{R l_{k}^{3}} l^{4}
$$

From the point of view of the problem, the case is most favorable $C=6$, for which angle $\Theta\left(l_{k}\right)$ is the largest and close to the corresponding value in the standard solution, where $\Theta\left(l_{k}\right)=\frac{1}{R} l_{k}$. For this case, the following dependencies apply:

- In the interval $l \in\left\langle 0, \frac{l_{k}}{2}\right\rangle$

$$
\begin{aligned}
& k(l)=\frac{6}{R l_{k}} l-\frac{12}{R l_{k}^{2}} l^{2}+\frac{8}{R l_{k}^{3}} l^{3} \\
& \Theta(l)=\frac{3}{R l_{k}} l^{2}-\frac{4}{R l_{k}^{2}} l^{3}+\frac{2}{R l_{k}^{3}} l^{4}
\end{aligned}
$$

- In the interval $l \in\left\langle\frac{l_{k}}{2}, l_{k}\right\rangle$

$$
\begin{gathered}
k(l)=\frac{2}{R}-\frac{6}{R l_{k}} l+\frac{12}{R l_{k}^{2}} l^{2}-\frac{8}{R l_{k}^{3}} l^{3} \\
\Theta(l)=-\frac{1}{4 R} l_{k}+\frac{2}{R} l-\frac{3}{R l_{k}} l^{2}+\frac{4}{R l_{k}^{2}} l^{3}-\frac{2}{R l_{k}^{3}} l^{4}
\end{gathered}
$$

Because for $C=6$, while maintaining the existing slant, the angle of incline $\Theta\left(l_{k}\right)=$ $\frac{3}{4 R} l_{k}$, required reverse track length is

$$
l_{k}=\frac{4}{3} R \operatorname{atan} \frac{1}{n}
$$

Figure 4 shows the graph of nonlinear curvature described by the class function $C^{0}$ (for coefficient $\mathrm{C}=6$ ) along the entire length of the reverse turnout $\mathrm{Rz} 1: 14-760$. Applying this solution would require extension of the reverse track to $l_{k}=72,258 \mathrm{~m}$. 
The unequal horizontal axis of the feedback path is described by the corresponding parametric equations. For further consideration, a solution for the coefficient was adopted $\mathrm{C}=$ 6.

- The interval $l \in\left\langle 0, \frac{l_{k}}{2}\right\rangle$

$$
\begin{gathered}
x(l)=l-\frac{9}{10 R^{2} l_{k}^{2}} l^{5}+\frac{2}{R^{2} l_{k}^{3}} l^{6}-\frac{2}{R^{2} l_{k}^{4}} l^{7}+\frac{1}{R^{2} l_{k}^{5}} l^{8} \\
y(l)=\frac{1}{R l_{k}} l^{3}-\frac{1}{R l_{k}^{2}} l^{4}+\frac{2}{5 R l_{k}^{3}} l^{5}
\end{gathered}
$$

- The interval $l \in\left\langle\frac{l_{k}}{2}, l_{k}\right\rangle$

$x(l)=x\left(\frac{l_{k}}{2}\right)+\cos \left(\frac{3 l_{k}}{8 R}\right)\left(l-l_{20}\right)-\frac{1}{2 R} \sin \left(\frac{3 l_{k}}{8 R}\right)\left(l-l_{20}\right)^{2}-\frac{1}{6 R^{2}} \cos \left(\frac{3 l_{k}}{8 R}\right)\left(l-l_{20}\right)^{3}$

$y(l)=y\left(\frac{l_{k}}{2}\right)+\sin \left(\frac{3 l_{k}}{8 R}\right)\left(l-\frac{l_{k}}{2}\right)+\frac{1}{2 R} \cos \left(\frac{3 l_{k}}{8 R}\right)\left(l-\frac{l_{k}}{2}\right)^{2}-\frac{1}{6 R^{2}} \sin \left(\frac{3 l_{k}}{8 R}\right)\left(l-\frac{l_{k}}{2}\right)^{3}$

Diagram of the function $\mathrm{y}(\mathrm{x})$ for the reverse turnout $\mathrm{Rz}$ 1:14-760 nonlinear curvature described by the class function $\mathrm{C}^{0}$ shown in Figure 5. The length of the travel would have to rise to $a=72,280 \mathrm{~m}$.

Necessity of turnout extension at $\frac{1}{3}$ It is still a lot but much less than in the previous two cases. Of course, the standard length of the system could be retained, but this would have reduced the radius of the circular arc about $25 \%$ (or correct the turnout slant).

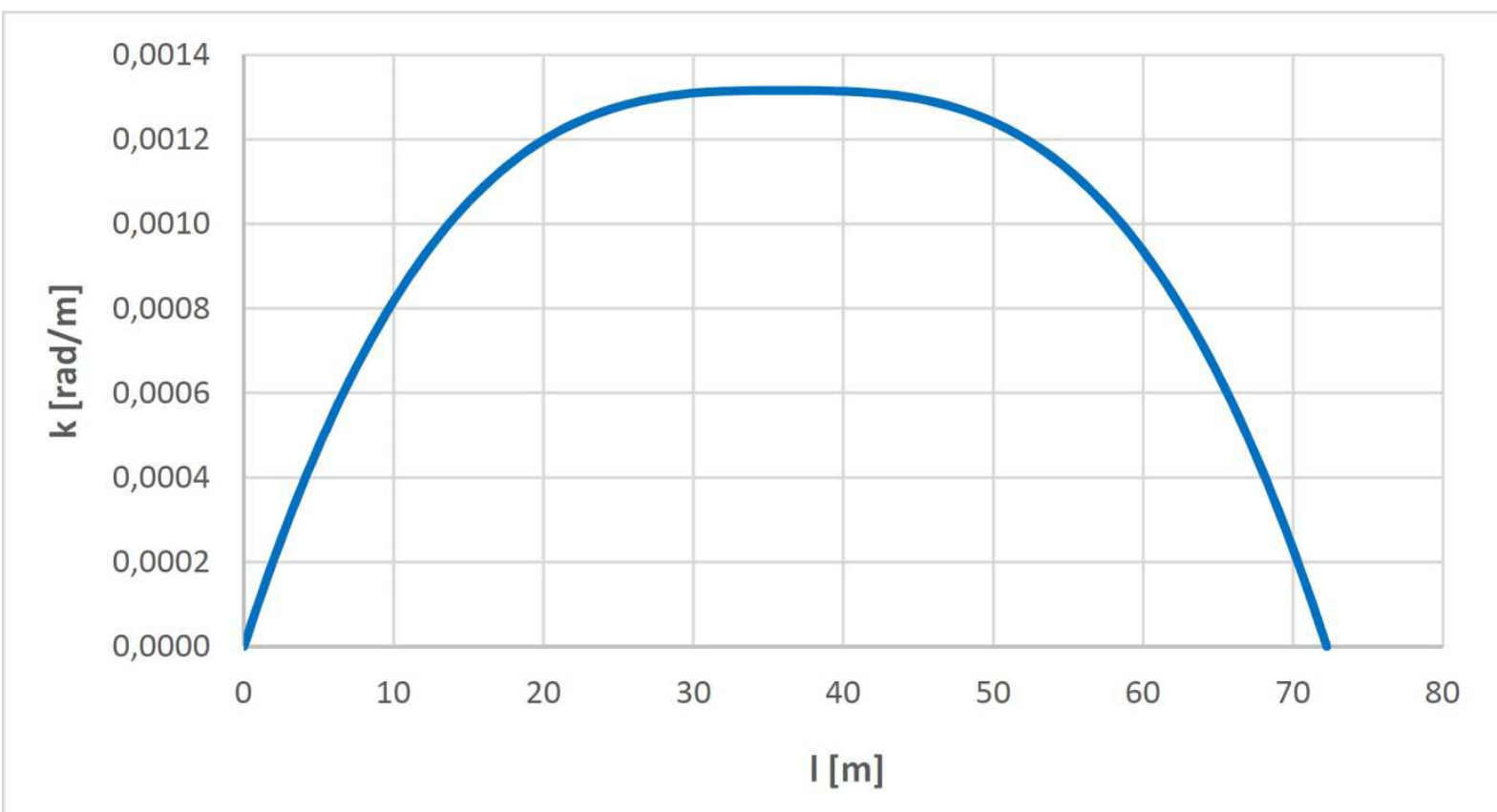

4. Graph of non-linear curves described by the class function $C^{0}$ (for $C=6$ ) along the entire length of the reverse turnout track $\mathrm{Rz} 1: 14-760$ 


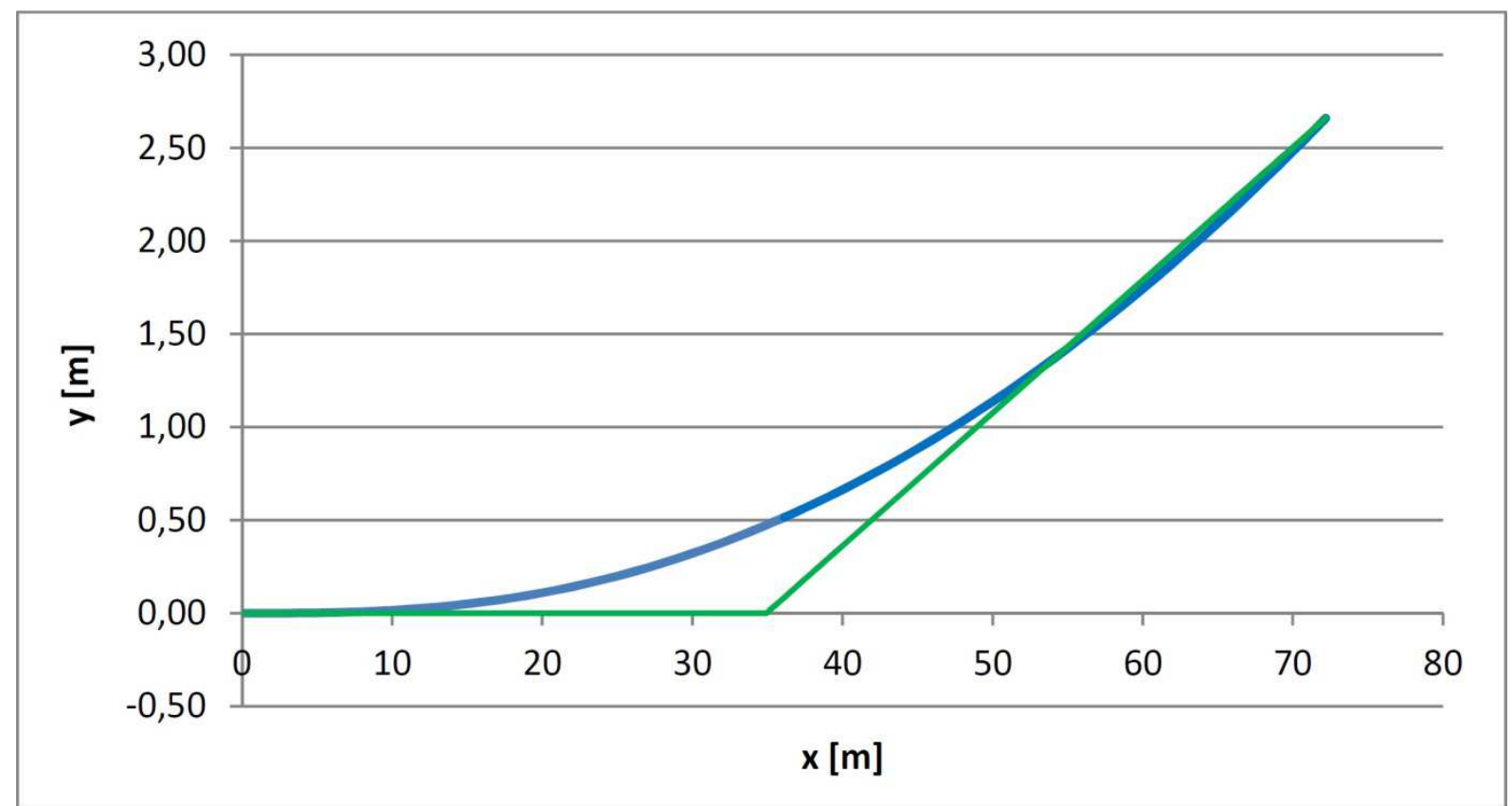

5. Graph of the function $y(x)$ for the reverse turnout track Rz 1:14-760 nonlinear curvature described by the class function $\mathrm{C}^{0}$ (on a contaminated scale)

\section{Possibilities of practical use of obtained solutions}

Applying a variable curvature on the length of the entire turnout (in the reverse track), with its chamfer and arc radius, necessitates a significant increase in the length of the turn. However, this problem applies in principle to separated turnouts. In parallel tracks, the basic issue is to keep track gauge in a good order. The final turn of the track is the decisive role that can be shaped by manipulating the pitch and the travel radius. The analytical record gives a very wide range of possibilities.

Solutions were carried out with a linear curvature over the entire length of the reverse track (Variant I) and the nonlinear curvature described by the class function $\mathrm{C}^{0}$ for coefficient $C=6$ (Wariant III). By changing the slant and the turnout radius (of which stemmed its length $l_{k}$ ) was sought to obtain a final ordinate $y\left(l_{k}\right)=2 \mathrm{~m}$ (half of the typical gait track on the trails).

Table 1 shows the results obtained in the search for a suitable solution for variant I and in table 2 for variant III. In both cases, the starting point was $\mathrm{Rz} 1: 14-760$, where the radius $R$ was first changed keeping the slant $1: n$, Then the bevel was changed to the radius, and finally the bevel and the radius were changed. The exact value of the final ordinate was obtained by taking the fractional part into the slope. Figures $\mathbf{6}$ and $\mathbf{7}$ show all endpoint solutions, based on tables $\mathbf{1}$ and $\mathbf{2}$. 


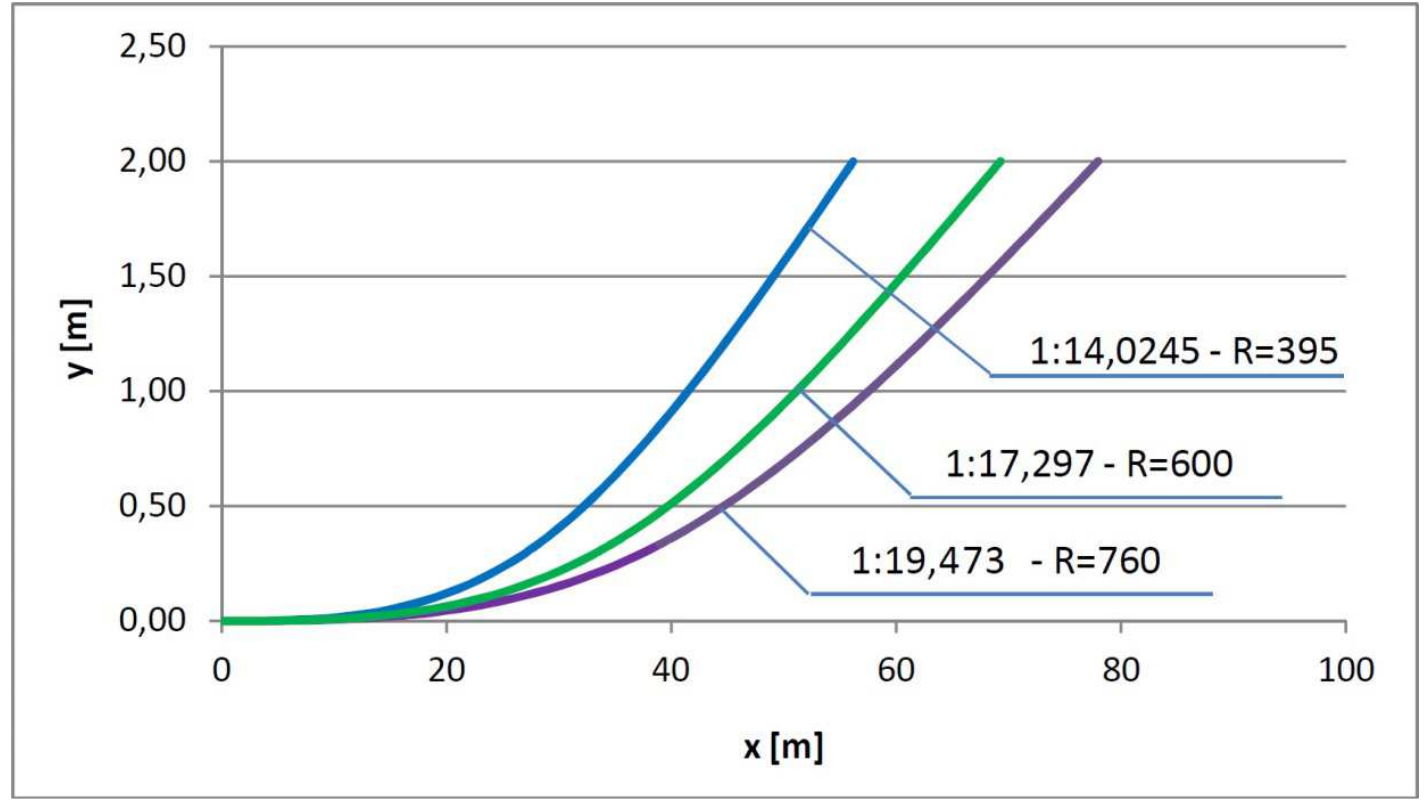

6. Diagrams of function $\mathrm{y}(\mathrm{x})$ for the return path at the assumed value of the ordinal (based on Table 1, in the contaminated scale)

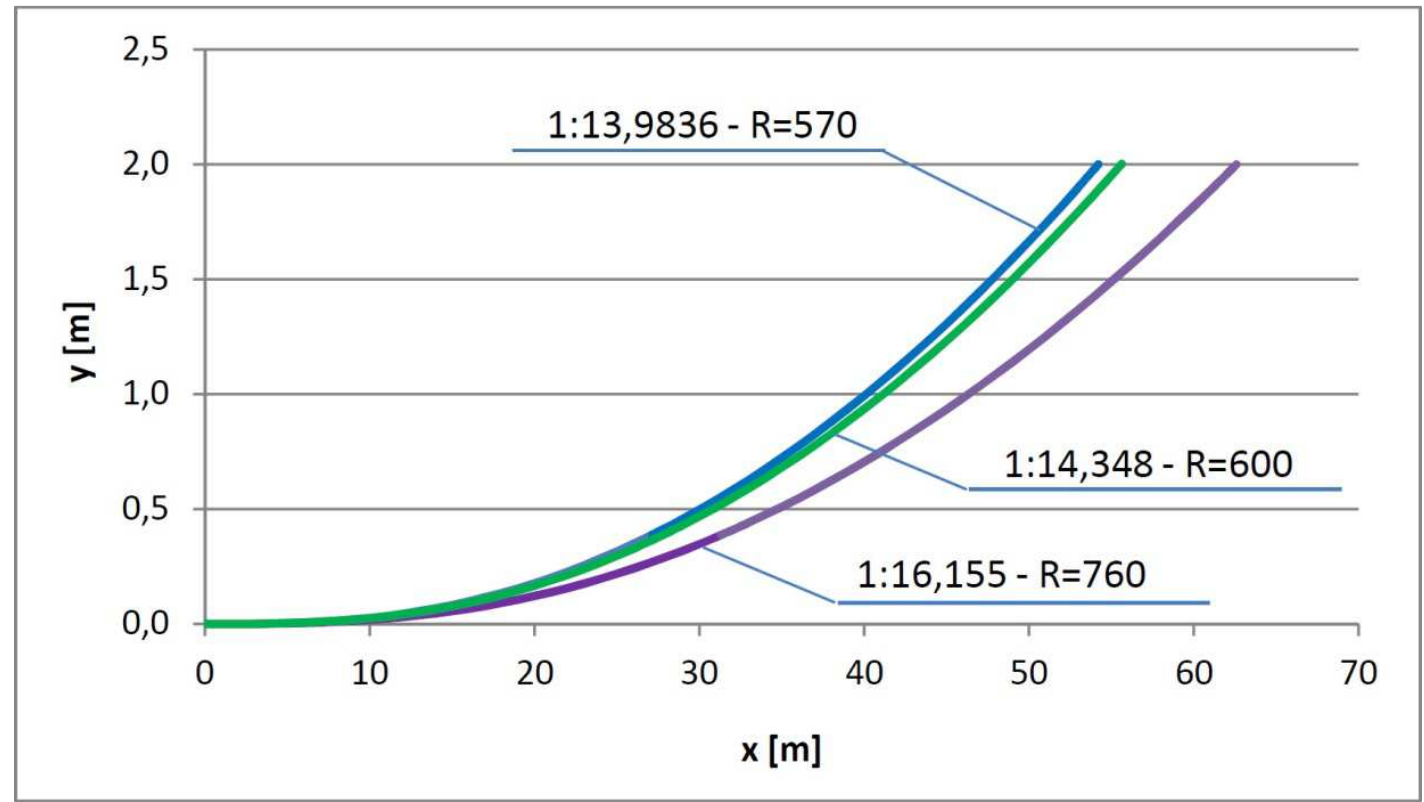

7. Diagrams of function $\mathrm{y}(\mathrm{x})$ for the return path at the assumed value of the ordinate (based on Table 2 , in the contaminated scale)

Tab. 1. Selected results obtained during the search for a suitable solution for the variant I

\begin{tabular}{|c|c|c|c|}
\hline Radius $R[\mathrm{~m}]$ & Turnout slant $n$ & $\begin{array}{c}\text { Length of the reverse } \\
\text { track } l_{k}\end{array}$ & Final ordinate $y\left(l_{k}\right)$ \\
\hline 760 & 14 & 108,387 & 3,862 \\
500 & 14 & 71,307 & 2,541 \\
400 & 14 & 57,046 & 2,032 \\
395 & 14 & 56,333 & 2,007 \\
395 & 14,0245 & 56,235 & 2,000 \\
\hline
\end{tabular}




\begin{tabular}{|l|l|l|l|}
\hline 760 & 17 & 89,309 & 2,622 \\
760 & 20 & 75,937 & 1,896 \\
760 & 19 & 79,926 & 2,101 \\
760 & 19,5 & 77,870 & 1,994 \\
760 & 19,473 & 77,988 & 2,000 \\
\hline 600 & 16 & 74,903 & 2,336 \\
600 & 17 & 70,507 & 2,070 \\
600 & 17,2 & 69,689 & 2,023 \\
600 & 17,3 & 69,287 & 1,999 \\
600 & 17,297 & 69,299 & 2,000 \\
\hline
\end{tabular}

Tab. 2. Selected results obtained during the search for a suitable solution for the variant III

\begin{tabular}{|c|c|c|c|}
\hline Radius $R[\mathrm{~m}]$ & Turnout slant $n$ & $\begin{array}{c}\text { Length of the reverse } \\
\text { track } l_{k}\end{array}$ & Final ordinate $y\left(l_{k}\right)$ \\
\hline 760 & 14 & 72,258 & 2,660 \\
600 & 14 & 57,046 & 2,100 \\
550 & 14 & 52,292 & 1,925 \\
570 & 14 & 54,194 & 1,995 \\
570 & 13,9836 & 54,257 & 2,000 \\
\hline 760 & 15 & 67,456 & 2,319 \\
760 & 15,5 & 65,286 & 2,172 \\
760 & 16 & 63,251 & 2,039 \\
760 & 16,1 & 62,859 & 2,014 \\
760 & 16,155 & 62,646 & 2,000 \\
\hline 600 & 15 & 53,255 & 1,831 \\
600 & 14,5 & 55,085 & 1,959 \\
600 & 14,4 & 55,467 & 1,986 \\
600 & 14,3 & 55,853 & 2,014 \\
600 & 14,348 & 55,667 & 2,000 \\
\hline
\end{tabular}

From the data presented it follows that the required travel distance in variant III is considerably lower than in variant I. It should be noted here that the solution obtained in variant $\mathrm{I}$ by decreasing the radius itself $R$ it is characterized by a radical reduction of train speed. In both variants the same increase $n$ This leads to a significant extension of the turnout. For variant I simultaneous reduction $R$ and increasing $n$ is the only way to solve the problem.

\section{Summary}

In the typical railroad used from the start of the railway, a single circular arc without transition curves is used. As a result, there are places of violent, jumping change of the ordinate curvature at the beginning and end of turnout. In recent years, in many countries, in order to smooth the curvature of these regions, the "Clitoris sections" on both sides of the circular arc are brought on, on which the curvature varies linearly.

In the case of standard turnouts, the change of the run-to-go radius of the larger radius in the reverse track usually results in the need to increase the track gauge. In the case of turnouts with variable curvature - due to the individual nature of their design - it is possible to adjust the given turnout to local requirements. This is especially important on parallel tracks, where the basic issue is to maintain a proper track gauge. The final ordinate of the track is decisive here. 
The paper presents three variants of the problem solution, characterized by variable curvature over the entire length of the reverse turnout track. The case of linear curvature and two cases of nonlinear curvature were described: class function $\mathrm{C}^{1}$ and the class function $\mathrm{C}^{0}$. As it turned out, the use of both linear curvature, as well as the described function of class $\mathrm{C} 1$, leads to the necessity of a significant (even twice) extension of the reverse track in relation to the base turnout. The curvature described by the class function $\mathrm{C}^{0} \mathrm{In}$ this respect, it has been considerably more beneficial, although the necessary extension of turnout is still significant. In this situation, one would have to consider using a variable curvature in a limited manner, leaving a segment of the circular arc over the length of the reverse turnout track . In turning with a variable curvature of the reverse track, it is possible to flexibly shape the horizontal ordinates by manipulating the chamfer and the turnout radius. The paper presents the effects of such a procedure, which was aimed at obtaining the appropriate value of the final ordinate.

\section{Source materials}

[1] Koc W.: Analytical method of modelling the geometric system of communication route. Mathematical Problems in Engineering, vol. 2014, Article ID 679817, 2014.

[2] Nowakowski M.J.: Rozjazdy do dużych prędkości o zmiennej krzywiźnie toru zwrotnego. Przegląd Komunikacyjny 2011, nr 9-10, s. 88-91.

[3] Omieczyński P.: Analiza oraz wykorzystanie rozjazdów o zmiennej krzywiźnie toru zwrotnego. Praca dyplomowa, Politechnika Gdańska 2016.

[4] Plank B.: Linie dużych prędkości realizowane przez VAE. Prezentacja firmy Voestalpine GmbH, 2007.

[5] Technical Memorandum: Alignment design standards for high-speed train operation. Prepared by Parsons Brinckerhoff for the California High-Speed Rail Authority, USA, 2009.

[6] Wang P.: Design of high-speed railway turnouts. Theory and Applications. Academic Press, Published by Elsevier Inc., 2015. 